\title{
Perioperative Patient Factors Related to 2-Year Outcome after Esophageal Cancer Surgery: A Retrospective Cohort Study
}

\author{
Maiko Hasegawa-Moriyama1,2* ${ }^{\text {, Yuichi Kanmura1 }}$ \\ ${ }^{1}$ Department of Anesthesiology and Critical Care Medicine, Graduate School of Medical and Dental Sciences, \\ Kagoshima University, Sakuragaoka, Kagoshima, Japan \\ ${ }^{2}$ Operating Suite, Shinshu University Hospital, Asahi, Matsumoto, Nagano, Japan \\ Email: `hasegawa-moriyama@shinshu-u.ac.jp, kanmura@m3.kufm.kagoshima-u.ac.jp
}

How to cite this paper: Hasegawa-Moriyama, M. and Kanmura, Y. (2020) Perioperative Patient Factors Related to 2-Year Outcome after Esophageal Cancer Surgery: A Retrospective Cohort Study. Open Journal of Anesthesiology, 10, 101-112.

https://doi.org/10.4236/ojanes.2020.104009

Received: March 23, 2020

Accepted: April 6, 2020

Published: April 9, 2020

Copyright $\odot 2020$ by author(s) and Scientific Research Publishing Inc. This work is licensed under the Creative Commons Attribution International License (CC BY 4.0).

http://creativecommons.org/licenses/by/4.0/

\begin{abstract}
Background: Surgical treatment for esophageal cancer is major invasive surgery. Recently, opioids have been implicated in the promotion of cancer recurrence and metastasis because of their immunosuppressive effects. However, it has not been fully evaluated whether the intraoperative use of opioids is directly correlated with postoperative prognosis in the clinical setting. Therefore, this study aimed to identify perioperative factors related to postoperative complications and 2-year survival in patients undergoing esophageal cancer surgery. Methods: We retrospectively reviewed 105 patients who underwent surgery for esophageal cancer at Kagoshima University Hospital from January 2013 to December 2017. Patient parameters were compared between survivors and non-survivors for 2 years after surgery. Results: The 2-year survival rate after esophageal cancer surgery was $76.2 \%$ (80 survivors vs. 25 non-survivors). The incidence of postoperative pneumonia within 7 days after surgery was significantly higher in non-survivors compared with survivors (36.0\% vs. $16.3 \%$; odds ratio, 2.90 ; $95 \%$ confidence interval, $1.06-7.96, P<$ 0.05). Pneumonia was associated with postoperative metastasis (odds ratio, 3.00; 95\% confidence interval, $1.117-8.072, P=0.029)$. The intraoperative dosage of opioids calculated as fentanyl equivalents was not correlated with any postoperative complications or 2-year survival. The preoperative albumin level and neutrophil-lymphocyte ratio were associated with the incidence of postoperative pneumonia. Conclusions: Postoperative pneumonia may be a possible predictor of 2-year prognosis in patients after esophageal cancer surgery, and is not correlated with intraoperative use of opioids. Preoperative physical status including immune and nutrient states may be more important for postoperative prognosis after esophageal cancer surgery.
\end{abstract}




\section{Keywords}

Esophageal Cancer, Prognosis, Opioids

\section{Introduction}

Esophageal cancer was estimated to be responsible for 1 in every 20 cancer deaths in 2018 worldwide [1]. Surgical treatment for esophageal cancer is major invasive surgery that is frequently accompanied by postoperative complications such as anastomotic leakage and pneumonia [2]. Patients who underwent esophageal cancer surgery had a 5-year overall recurrence-free survival rate of $36.4 \%$ and 5-year overall recurrence rate of $60 \%$ [3], revealing a high rate of recurrence even after surgery. A high incidence of distant metastasis remains a long-term problem because the surgical procedure itself can spread circulating tumor cells and lead to subsequent metastasis after esophagectomy [4]. Thus, the perioperative impairment of the immune state arising from surgical stress is linked to cancer recurrence. In a previous study, the overall 1-year and 2-year post-recurrence survival rates in patients undergoing gastric cancer surgery were $32.6 \%$ and $12.6 \%$, respectively, with a median survival after recurrence of 6.0 months [5]. Therefore, neoadjuvant chemoradiotherapy is frequently performed depending on patient physical status and cancer progression before or after esophageal cancer surgery. Although neoadjuvant therapy has improved the 5-year progression-free survival rate to $44 \%$, the prognosis for esophageal cancer remains poor compared with that for other gastrointestinal cancers [6]. However, although neoadjuvant chemoradiotherapy does not affect perioperative immune function in neoadjuvant chemoradiotherapy-sensitive patients, it may significantly reduce this function in neoadjuvant chemoradiotherapy-insensitive patients [7].

Patients undergoing esophageal cancer surgery with three-field lymph node dissection require analgesia comprising high-dose usage of opioids. Intraoperative factors including surgical stress [8], blood transfusion [9], opioids [10], and general anesthesia [11] have been implicated in immune function impairment in the perioperative period. Meanwhile, intravenous anesthesia with propofol during esophageal cancer surgery was associated with better postoperative survival rates than volatile anesthesia with isoflurane, sevoflurane, and desflurane [12]. Furthermore, systemic reviews revealed that regional anesthesia had some modulatory effects on inflammatory and immunological responses in patients although there is no evidence to support or refute the use of epidural anesthesia to reduce cancer recurrence after gastroesophageal cancer surgery [13]. Although the correlation between intraoperative opioid use and survival has not been clarified, postoperative opioid use was recently demonstrated not to be associated with survival and recurrence-free survival after esophageal cancer surgery, while postoperative complications may increase the hazard ratio for survival and recurrence-free survival [14]. Despite the enormous number of in vitro and animal studies indicating the immunosuppressive effects of opioids, it currently remains 
unclear whether opioids augment the risk of recurrence in clinical practice [15].

Therefore, this study aimed to identify the perioperative factors (including opioid administration) related to postoperative adverse events and prognosis in patients undergoing esophageal cancer surgery.

\section{Methods}

We retrospectively reviewed 105 patients who underwent esophagectomy with three-field lymph node dissection at Kagoshima University Hospital from January 2013 to February 2017. The study protocol was reviewed and approved by the local ethics committee of Kagoshima University. Patients who were not followed up for 2 years after surgery were excluded from the present study. Medical records including anesthesia charts were reviewed to evaluate the perioperative factors related to the 2-year prognosis after esophageal cancer surgery.

Opioids were not preoperatively prescribed to any patients. An epidural catheter was inserted at the level of Th9/10. In all patients, anesthesia was induced with propofol, remifentanil, and rocuronium. Anesthesia was maintained by continuous intravenous target-controlled infusion of propofol with an effect-site concentration of $2.5-4.0 \mu \mathrm{g} / \mathrm{mL}$ to keep the bispectral index between 40 and 60 . During surgery, remifentanil was administered at a rate of $0.2-1.0 \mu \mathrm{g} / \mathrm{kg} / \mathrm{min}$. Fentanyl was intermittently administered through an intravenous line. A single injection of $1-3 \mathrm{mg}$ morphine was performed through the epidural catheter in some patients. The total dose of opioids including remifentanil, fentanyl, and morphine was expressed by fentanyl equivalents (FE) as previously described [16]. Rocuronium was administered based on train-of-four monitoring. Sedation with dexmedetomidine $(0.4-0.7 \mathrm{~g} / \mathrm{kg} / \mathrm{h})$ and propofol $(3.0-4.0 \mathrm{mg} / \mathrm{kg} / \mathrm{h})$ was continued from the end of surgery until postoperative day 1 .

White blood cell, neutrophil, and lymphocyte counts were performed between the induction of anesthesia and start of surgery (preoperative) and at the end of anesthesia (postoperative) using an automatic blood cell counter (KX-21; Sysmex, Kobe, Japan).

Parameters were compared between 2-year survivors and non-survivors. The Mann-Whitney $U$-test was used for the comparison of numerical data. The Fisher exact test was used to identify differences in expected frequency versus observed frequency of nominal variables. Factors correlated with 2-year survival, metastasis, and pneumonia were analyzed by multiple logistic regression analysis. Data are presented as median (interquartile range). A value of $P<0.05$ was considered statistically significant. All statistical analyses were performed using SPSS version 24 software (IBM Corp., Armonk, NY).

\section{Results}

Among the 105 patients undergoing esophageal cancer surgery with three-field lymph node dissection, the 2-year survival rate was $76.2 \%$ (80 survivors vs. 25 non-survivors) (Table 1). There were no differences between survivors and non-survivors in patient characteristics including age, body mass index, American 
Table 1. Demographic characteristics of patients undergoing surgery for esophageal cancer.

\begin{tabular}{|c|c|c|c|c|c|}
\hline Variable & Levels & Total & $\begin{array}{l}\text { Survivors } \\
(\mathrm{n}=80)\end{array}$ & $\begin{array}{l}\text { Non-survivors } \\
\quad(\mathrm{n}=25)\end{array}$ & $P$-value \\
\hline \multirow{4}{*}{$\begin{array}{c}\text { Survival after } \\
\text { surgery }\end{array}$} & $>2$ years & 80 & 80 & 0 & - \\
\hline & & & & & \\
\hline & $1-2$ years & 17 & 0 & 17 & - \\
\hline & $<1$ year & 8 & 0 & 8 & - \\
\hline Age (y) & & $65(61-70)$ & $65(61-70)$ & $66(60-70)$ & 0.95 \\
\hline \multirow{2}{*}{ Sex } & Male & 89 & 69 & 20 & 0.52 \\
\hline & Female & 16 & 11 & 5 & \\
\hline \multirow{2}{*}{ ASA } & 1 or 2 & 102 & 79 & 23 & 0.14 \\
\hline & 3 or 4 & 3 & 1 & 2 & \\
\hline \multirow{3}{*}{ Tumor stage } & I & 21 & 19 & 2 & 0.07 \\
\hline & II & 24 & 20 & 4 & \\
\hline & III & 60 & 41 & 19 & \\
\hline \multirow{2}{*}{ Histology } & SCC & 101 & 77 & 24 & 1.00 \\
\hline & Adenocarcinoma & 4 & 3 & 1 & \\
\hline \multirow{2}{*}{ Type of surgery } & Open thoracotomy & 89 & 66 & 23 & 0.34 \\
\hline & Thoracoscopy & 16 & 14 & 2 & \\
\hline Multiple cancer & Yes/No & $21 / 84$ & $18 / 62$ & $3 / 22$ & 0.39 \\
\hline $\begin{array}{l}\text { Neoadjuvant } \\
\text { chemotherapy }\end{array}$ & Yes/No & $79 / 26$ & $57 / 23$ & $22 / 3$ & 0.11 \\
\hline $\begin{array}{l}\text { Neoadjuvant } \\
\text { radiation }\end{array}$ & Yes/No & $55 / 50$ & $41 / 39$ & $14 / / 11$ & 0.81 \\
\hline Body mass index & & $\begin{array}{c}22.4 \\
(19.8-25.6)\end{array}$ & $\begin{array}{c}22.5 \\
(20.0-26.7)\end{array}$ & $\begin{array}{c}21.4 \\
(19.2-24.3)\end{array}$ & 0.26 \\
\hline smoking & Yes/No & $86 / 19$ & $66 / 14$ & $20 / 5$ & 0.77 \\
\hline COPD & Yes/No & $12 / 93$ & $8 / 72$ & $4 / 21$ & 0.47 \\
\hline Diabetes & Yes/No & $14 / 91$ & $12 / 68$ & $2 / 23$ & 0.51 \\
\hline Hypertension & Yes/No & $37 / 68$ & $28 / 52$ & $9 / 16$ & 1.00 \\
\hline Renal disease & Yes/No & $4 / 101$ & $3 / 77$ & $1 / 24$ & 1.00 \\
\hline $\begin{array}{l}\text { Plasma albumin } \\
\qquad(\mathrm{mg} / \mathrm{dL})\end{array}$ & & $\begin{array}{c}3.9 \\
(3.7-4.3)\end{array}$ & $\begin{array}{c}3.9 \\
(3.7-4.3)\end{array}$ & $\begin{array}{c}3.6 \\
(3.3-3.9)\end{array}$ & $<0.01$ \\
\hline $\begin{array}{c}\text { Plasma CRP } \\
(\mathrm{mg} / \mathrm{dL})\end{array}$ & & $\begin{array}{c}0.08 \\
(0.03-0.31)\end{array}$ & $\begin{array}{c}0.07 \\
(0.03-0.3)\end{array}$ & $\begin{array}{c}0.13 \\
(0.05-0.47)\end{array}$ & 0.13 \\
\hline
\end{tabular}

SCC: squamous cell carcinoma; COPD: chronic obstructive pulmonary disease; CRP: C-reactive protein.

Society of Anesthesiologists classification, or preoperative complications such as diabetes. However, the albumin level within 2 weeks before surgery was significantly lower in non-survivors than in survivors $(P<0.01)$. Similarly, preoperative and postoperative levels of hemoglobin and hematocrit were significantly lower in non-survivors compared with survivors (Table 2). Consistent with the 
lack of difference in blood loss between survivors and non-survivors, neither volume of total infusion nor blood transfusion differed between the two groups. However, the preoperative plasma glucose level was lower in non-survivors compared with survivors. Meanwhile, the detection rate of lymph node metastasis evaluated by intraoperative rapid pathological diagnosis was higher in non-survivors than in survivors. There were no significant difference between survivors and non-survivors regarding the total intraoperative dose of fentanyl and remifentanil, epidural use of morphine, or intraoperative total amount of opioids expressed as FE. Consistently, multiple logistic regression analysis revealed that FE was not correlated with postoperative complications including surgical site infection (SSI), anastomotic leakage, or pneumonia as well as metastasis or survival within 2 years after surgery (Table 3 ). The incidence of postoperative pneumonia within 7 days after surgery was significantly higher in non-survivors compared with survivors (36.0\% vs. $16.3 \%$; odds ratio [OR], $2.90 ; 95 \%$ confidence interval [CI], 1.06 - 7.96; $P<0.05)$. Compared with survivors, non-survivors had higher risks of pneumonia (OR, 2.90; $P<0.05$ ), metastasis within 2 years (OR, 7.11; $P<0.001$ ), and requirement for postoperative chemotherapy (OR, 3.48; $P=0.01)$. Consistent with the correlation between postoperative pneumonia and 2-year survival, postoperative pneumonia was associated with metastasis within 2 years (adjusted OR, 3.003; 95\% CI, $1.117-8.072 ; P=0.029$ ) (Table 4). Moreover, the risk factors for postoperative pneumonia were preoperative low plasma albumin level (adjusted OR, 0.189; 95\% CI, $0.047-0.759 ; P=0.02$ ) and high neutrophil-lymphocyte ratio (adjusted OR, 1.229; 95\% CI, $1.035-1.460 ; P=0.02$ ).

\section{Discussion}

In this study, no significant correlations between prognosis after esophageal cancer surgery and intraoperative factors including anesthesia or opioid use were detected, although there were significant differences between 2-year survivors and non-survivors regarding the albumin level within 2 weeks before surgery, glucose level before the start of surgery, and both hemoglobin and hematocrit before and after surgery (Table 1 and Table 2). Furthermore, lymph node metastasis was less frequently detected by intraoperative rapid pathological diagnosis in 2-year survivors. These results suggest that the preoperative physical status related to tumor progression stage may have more influence on the postoperative prognosis (including cancer recurrence) than intraoperative events in patients with low physical status undergoing highly invasive surgery.

Perioperative stress can lead to a systemically immunocompromised condition associated with reduced lymphocyte numbers and expression of human leukocyte antigen-antigen D-related protein on lymphocytes and monocytes [17], resulting in susceptibility to postoperative infection and tumor progression [15]. An elevated neutrophil/lymphocyte ratio is reportedly to be correlated with the incidence of SSI after posterior lumbar instrumentation surgery [18] and cardiac surgery [19], while the perioperative neutrophil-lymphocyte ratio is correlated 
Table 2. Patient data during anesthesia for esophageal cancer surgery.

\begin{tabular}{|c|c|c|c|c|c|}
\hline Variable & & Total & $\begin{array}{l}\text { Survivors } \\
(\mathrm{n}=80)\end{array}$ & $\begin{array}{c}\text { Non-survivors } \\
\quad(\mathrm{n}=25)\end{array}$ & $P$-value \\
\hline Anesthesia time (min) & & $\begin{array}{c}693 \\
(634-738)\end{array}$ & $\begin{array}{c}708 \\
(629-740)\end{array}$ & $\begin{array}{c}683 \\
(638-731)\end{array}$ & 0.31 \\
\hline Operation time $(\mathrm{min})$ & & $\begin{array}{c}583 \\
(513-619)\end{array}$ & $\begin{array}{c}586 \\
(517-626)\end{array}$ & $\begin{array}{c}581 \\
(496-603)\end{array}$ & 0.24 \\
\hline \multirow{2}{*}{ WBC counts $(/ \mu \mathrm{L})$} & Pre & $\begin{array}{c}3800 \\
(3000-4900)\end{array}$ & $\begin{array}{c}3750 \\
(2825-4700)\end{array}$ & $\begin{array}{c}4200 \\
(3500-5350)\end{array}$ & 0.08 \\
\hline & Post & $\begin{array}{c}8100 \\
(6500-10,700)\end{array}$ & $\begin{array}{c}8200 \\
(6525-10,775)\end{array}$ & $\begin{array}{c}8100 \\
(5850-9900)\end{array}$ & 0.72 \\
\hline \multirow{2}{*}{$\begin{array}{l}\text { Neutrophil counts } \\
(/ \mu \mathrm{L})\end{array}$} & Pre & $\begin{array}{c}2400 \\
(1800-3000)\end{array}$ & $\begin{array}{c}2300 \\
(1700-3000)\end{array}$ & $\begin{array}{c}2500 \\
(2150-3800)\end{array}$ & 0.08 \\
\hline & Post & $\begin{array}{c}7200 \\
(5250-9500)\end{array}$ & $\begin{array}{c}7150 \\
(5400-9825)\end{array}$ & $\begin{array}{c}7200 \\
(5100-8750)\end{array}$ & 0.08 \\
\hline \multirow{2}{*}{$\begin{array}{l}\text { Lymphocyte counts } \\
\qquad(/ \mu \mathrm{L})\end{array}$} & Pre & $\begin{array}{c}900 \\
(600-1300)\end{array}$ & $\begin{array}{c}900 \\
(600-1200)\end{array}$ & $\begin{array}{c}1000 \\
(550-1450)\end{array}$ & 0.29 \\
\hline & Post & $\begin{array}{c}500 \\
(400-650)\end{array}$ & $\begin{array}{c}500 \\
(400-700)\end{array}$ & $\begin{array}{c}500 \\
(400-600)\end{array}$ & 0.67 \\
\hline \multirow{2}{*}{$\begin{array}{l}\text { Platelet counts } \\
\qquad\left(\times 10^{4} / \mu \mathrm{L}\right)\end{array}$} & Pre & $\begin{array}{c}16.9 \\
(13.4-20.0)\end{array}$ & $\begin{array}{c}15.9 \\
(13.3-19.1)\end{array}$ & $\begin{array}{c}17.9 \\
(14.1-3.2)\end{array}$ & 0.14 \\
\hline & Post & $\begin{array}{c}15.0 \\
(12.2-18.7)\end{array}$ & $\begin{array}{c}15.2 \\
(12.0-18.7)\end{array}$ & $\begin{array}{c}15.0 \\
(13.1-19.9)\end{array}$ & 0.42 \\
\hline \multirow{2}{*}{$\begin{array}{l}\text { Neutrophil-lymphocyte } \\
\text { ratio }\end{array}$} & Pre & $\begin{array}{c}2.4 \\
(1.8-4.3)\end{array}$ & $\begin{array}{c}2.32 \\
(1.78-3.97)\end{array}$ & $\begin{array}{c}2.88 \\
(1.79-4.79)\end{array}$ & 0.54 \\
\hline & Post & $\begin{array}{c}15.0 \\
(11.0-19.8)\end{array}$ & $\begin{array}{c}14.90 \\
(11.03-20.33)\end{array}$ & $\begin{array}{c}15.00 \\
(10.34-19.18)\end{array}$ & 0.94 \\
\hline \multirow{2}{*}{$\begin{array}{l}\text { Platelet-lymphocyte } \\
\text { ratio }\end{array}$} & Pre & $\begin{array}{c}180 \\
(133-272)\end{array}$ & $\begin{array}{c}181 \\
(132-265)\end{array}$ & $\begin{array}{c}175 \\
(135-298)\end{array}$ & 0.89 \\
\hline & Post & $\begin{array}{c}303 \\
(223-401)\end{array}$ & $\begin{array}{c}297 \\
(220-370)\end{array}$ & $\begin{array}{c}352 \\
(232-484)\end{array}$ & 0.14 \\
\hline \multirow{2}{*}{ Hemoglobin $(\mathrm{g} / \mathrm{dL})$} & Pre & $\begin{array}{c}10.0 \\
(9.2-11.0)\end{array}$ & $\begin{array}{c}10.3 \\
(9.2-11.2)\end{array}$ & $\begin{array}{c}9.3 \\
(9.0-10.4)\end{array}$ & 0.02 \\
\hline & Post & $\begin{array}{c}9.6 \\
(8.9-10.8)\end{array}$ & $\begin{array}{c}10.0 \\
(9.1-10.9)\end{array}$ & $\begin{array}{c}9.2 \\
(8.4-9.8)\end{array}$ & 0.008 \\
\hline \multirow{2}{*}{ Hematocrit (\%) } & Pre & $\begin{array}{c}30.4 \\
(27.6-32.6)\end{array}$ & $\begin{array}{c}30.7 \\
(27.6-33.5)\end{array}$ & $\begin{array}{c}28.5 \\
(27.0-31.0)\end{array}$ & 0.03 \\
\hline & Post & $\begin{array}{c}29.3 \\
(27.2-32.5)\end{array}$ & $\begin{array}{c}29.9 \\
(27.7-33.1)\end{array}$ & $\begin{array}{c}27.8 \\
(25.6-30.4)\end{array}$ & 0.007 \\
\hline \multirow{2}{*}{$\begin{array}{l}\text { Plasma glucose } \\
(\mathrm{mg} / \mathrm{dL})\end{array}$} & Pre & $\begin{array}{c}108.0 \\
(97.5-121.5)\end{array}$ & $\begin{array}{c}111 \\
(98.3-124.8)\end{array}$ & $\begin{array}{c}104.0 \\
(95.5-110.0)\end{array}$ & $<0.0001$ \\
\hline & Post & $\begin{array}{c}177 \\
(161-198)\end{array}$ & $\begin{array}{c}177 \\
(155-198)\end{array}$ & $\begin{array}{c}177 \\
(169-196)\end{array}$ & 0.47 \\
\hline \multirow{2}{*}{$\begin{array}{l}\text { Plasma lactate } \\
(\mathrm{mmol} / \mathrm{L})\end{array}$} & Pre & $\begin{array}{c}0.8 \\
(0.6-0.9)\end{array}$ & $\begin{array}{c}0.80 \\
(0.6-1.0)\end{array}$ & $\begin{array}{c}0.70 \\
(0.6-0.9)\end{array}$ & 0.10 \\
\hline & Post & $\begin{array}{c}2.2 \\
(1.6-2.8)\end{array}$ & $\begin{array}{c}2.2 \\
(1.6-2.7)\end{array}$ & $\begin{array}{c}2.3 \\
(1.5-3.2)\end{array}$ & 0.93 \\
\hline Blood loss (mL) & & $\begin{array}{c}290 \\
(203-493)\end{array}$ & $\begin{array}{c}280 \\
(183-428)\end{array}$ & $\begin{array}{c}410 \\
(218-585)\end{array}$ & 0.06 \\
\hline Infusion $(\mathrm{mL})$ & & $\begin{array}{c}4800 \\
(4035-5655)\end{array}$ & $\begin{array}{c}4900 \\
(4145-5800)\end{array}$ & $\begin{array}{c}4750 \\
(3905-5525)\end{array}$ & 0.38 \\
\hline
\end{tabular}




\section{Continued}

\begin{tabular}{|c|c|c|c|c|c|}
\hline $\begin{array}{l}\text { Blood transfusion } \\
\qquad(\mathrm{mL})\end{array}$ & & $0(0-0)$ & $\begin{array}{c}0 \\
(0-0)\end{array}$ & $0(0-0)$ & 0.99 \\
\hline Urine volume $(\mathrm{mL})$ & & $\begin{array}{c}155 \\
(968-2388)\end{array}$ & $\begin{array}{c}1573 \\
(1025-2455)\end{array}$ & $\begin{array}{c}1425 \\
(680-2310)\end{array}$ & 0.22 \\
\hline Balance $(\mathrm{mL})$ & & $\begin{array}{c}2680 \\
(2195-3448)\end{array}$ & $\begin{array}{c}2668 \\
(2216-3515)\end{array}$ & $\begin{array}{c}2680 \\
(2095-3305)\end{array}$ & 0.61 \\
\hline $\begin{array}{l}\text { Detection of lymph } \\
\text { node metastasis }\end{array}$ & Yes/No & $48 / 57$ & $31 / 49$ & $17 / 8$ & $<0.05$ \\
\hline $\begin{array}{l}\text { Total use of } \\
\text { remifentanil }(\mu \mathrm{g})\end{array}$ & & $\begin{array}{c}13,573 \\
(10,217-18,074)\end{array}$ & $\begin{array}{c}13,791 \\
(10,457-18,717)\end{array}$ & $\begin{array}{c}12,591 \\
(9096-17,161)\end{array}$ & 0.40 \\
\hline $\begin{array}{l}\text { Total use of } \\
\text { fentanyl }(\mu \mathrm{g})\end{array}$ & & $\begin{array}{c}800 \\
(600-1000)\end{array}$ & $\begin{array}{c}800 \\
(600-1000)\end{array}$ & $\begin{array}{c}800 \\
(600-860)\end{array}$ & 0.81 \\
\hline $\begin{array}{l}\text { Epidural use } \\
\text { of morphine }\end{array}$ & Yes/No & $68 / 37$ & $52 / 28$ & $16 / 9$ & 1.00 \\
\hline $\begin{array}{c}\text { Fentanyl equivalents } \\
\text { exchange }(\mu \mathrm{g})\end{array}$ & & $\begin{array}{c}14,303 \\
(10,949-18,924)\end{array}$ & $\begin{array}{c}14,529 \\
(11,566-19,559)\end{array}$ & $\begin{array}{c}13,390 \\
(9763-17,971)\end{array}$ & 0.37 \\
\hline
\end{tabular}

WBC: white blood cell; Pre: preoperative; Post: postoperative.

Table 3. Correlations between intraoperative opioid use and postoperative outcomes.

\begin{tabular}{ccccccccc}
\hline Variable & & $\begin{array}{c}\text { FE }<11,478 \\
(n=29)\end{array}$ & & $\begin{array}{c}\text { FE }>11,478 \\
(n=76)\end{array}$ & $\begin{array}{c}\text { Odds } \\
\text { ratio }\end{array}$ & 95\% CI & $P$-value \\
\hline $\begin{array}{c}\text { Surgical site } \\
\text { infection } \\
\begin{array}{c}\text { Anastomotic } \\
\text { leakage }\end{array}\end{array}$ & Yes/No & $5 / 24$ & $17.2 \%$ & $17 / 59$ & $22.4 \%$ & 0.72 & $0.24-2.18$ & 0.79 \\
$\begin{array}{c}\text { Pneumonia } \\
\text { Metastasis }\end{array}$ & Yes/No & $6 / 23$ & $20.7 \%$ & $15 / 61$ & $19.7 \%$ & 1.06 & $0.37-3.07$ & 1.0 \\
$\begin{array}{c}\text { within 2 years } \\
\text { Survival }\end{array}$ & Yes/No & $6 / 23$ & $20.7 \%$ & $15 / 61$ & $19.7 \%$ & 1.06 & $0.37-3.07$ & 1.0 \\
Yithin 2 years & Yes/No & $20 / 9$ & $68.9 \%$ & $60 / 16$ & $78.9 \%$ & 0.59 & $0.23-1.55$ & 0.31 \\
\hline
\end{tabular}

FE: fentanyl equivalents; CI: confidence interval.

Table 4. Postoperative outcomes in patients undergoing esophageal cancer surgery.

\begin{tabular}{|c|c|c|c|c|c|c|c|c|c|}
\hline Variable & & Total & $\begin{array}{c}\text { Survivors } \\
(n=80)\end{array}$ & & $\begin{array}{l}\text { Non-survivors } \\
\qquad(n=25)\end{array}$ & & $\begin{array}{l}\text { Odds } \\
\text { ratio }\end{array}$ & $95 \% \mathrm{CI}$ & $P$-value \\
\hline $\begin{array}{l}\text { Length of } \\
\text { stay (d) }\end{array}$ & & $\begin{array}{c}28 \\
(21-45)\end{array}$ & $\begin{array}{c}29 \\
(21-45)\end{array}$ & & $\begin{array}{c}27 \\
(21-41)\end{array}$ & & 0.99 & $0.97-1.01$ & 0.34 \\
\hline $\begin{array}{c}\text { Surgical site } \\
\text { infection }\end{array}$ & Yes/No & $23 / 82$ & $18 / 62$ & $22.5 \%$ & $5 / 20$ & $20.0 \%$ & 0.86 & $0.28-2.62$ & 1 \\
\hline $\begin{array}{c}\text { Anastomotic } \\
\text { leakage }\end{array}$ & Yes/No & $26 / 79$ & $21 / 59$ & $26.3 \%$ & $5 / 20$ & $20.0 \%$ & 0.70 & $0.23-2.1$ & 0.61 \\
\hline Pneumonia & Yes/No & $21 / 84$ & $13 / 67$ & $16.3 \%$ & $9 / 16$ & $36.0 \%$ & 2.90 & $1.06-7.96$ & $<0.05$ \\
\hline $\begin{array}{c}\text { Metastasis } \\
\text { within } 2 \text { years }\end{array}$ & Yes/No & $32 / 73$ & $16 / 64$ & $20 \%$ & $16 / 9$ & $64.0 \%$ & 7.111 & $2.66-19.02$ & $<0.001$ \\
\hline $\begin{array}{l}\text { Postoperative } \\
\text { chemotherapy }\end{array}$ & Yes/No & $32 / 73$ & $19 / 61$ & $23.8 \%$ & $13 / 12$ & $52.0 \%$ & 3.48 & $1.36-8.89$ & 0.01 \\
\hline $\begin{array}{l}\text { Postoperative } \\
\text { radiation }\end{array}$ & Yes/No & $12 / 93$ & $8 / 72$ & $10 \%$ & $4 / 21$ & $16.0 \%$ & 1.71 & $0.47-6.26$ & 0.47 \\
\hline
\end{tabular}

CI: confidence interval. 
with survival in patients undergoing colorectal cancer surgery [20] and higher mortality in patients with breast and kidney cancer [21]. In the present study, consistent with the lack of difference between survivors and non-survivors in the number of preoperative treatments involving neoadjuvant chemotherapy and radiation (Table 1), there were no difference between survivors and non-survivors in the neutrophil/lymphocyte ratio before the start of surgery or after the end of surgery (Table 2). Therefore, an immunosuppressed state, possibly related to nutrient state and tumor progression, may not be a single predictive factor for postoperative complications such as SSI, anastomotic leakage, pneumonia, and cancer recurrence.

A continuous infusion of remifentanil dose-dependently lowers the intraoperative release of stress hormones such as cortisol and catecholamine [22] that induce T-helper 2 cell dominance with modulation of cellular immunity [23]. Recently, opioid use, opioid potency, and the opioid dose were reported to be correlated with the risk of invasive pneumococcal disease induced by Streptococcus pneumoniae, although the potency of immunosuppression differed depending on the type of opioid [24]. The influence of intraoperative use of high doses of potent opioids, including remifentanil, fentanyl, and morphine, on postoperative infection and cancer progression after surgery has not been well elucidated. However, a concentration of remifentanil $(50 \mathrm{ng} / \mathrm{mL})$ similar to the relevant plasma concentration was shown to inhibit the migration of human polymorphonuclear neutrophils [25], and had weaker inhibitory effects on neutrophil migration than fentanyl $(30 \mathrm{ng} / \mathrm{mL})$. Furthermore, an 8-hour infusion of remifentanil did not affect natural killer cell activity in normal volunteers [26]. In our study, the total dose of opioids converted to FE was not correlated with the incidences of SSI, anastomotic leakage, pneumonia, metastasis, or survival within 2 years (Table 3). Although an evaluation of cancer immunity such as changes in natural killer cells or helper $\mathrm{T}$ cells was not performed, our findings suggest that the immunosuppressive effects of these opioids may be less clinically obvious in patients undergoing esophageal cancer surgery. The present study did not assess the postoperative use of opioids including those without immunosuppressive properties such as oxycodone, buprenorphine, and tramadol [15] [27]. Furthermore, the possibility that the use of opioids influenced the incidence of SSI cannot be excluded because of the small number of patients included in the present study. Further investigations are required to evaluate the correlations between the immunosuppressive effects of opioids and cancer prognosis.

The incidence of postoperative pneumonia was lower in 2-year survivors than in non-survivors (Table 5), consistent with the correlation between incidence of postoperative pneumonia within 7 days after surgery and metastasis within 2 years (Table 4). Preoperative albumin level and neutrophil-lymphocyte ratio were possible risk factors for occurrence of pneumonia (Table 6). Therefore, further evaluation is expected to clarify whether perioperative interventions for nutrient state and immune status can alter the postoperative prognosis in esophageal cancer patients. 
Table 5. Perioperative risk factors for metastasis within 2 years in patients undergoing esophageal cancer surgery.

\begin{tabular}{ccccccc}
\hline Variable & Unadjusted OR & $95 \%$ CI & P value & Adjusted OR & $95 \%$ CI & $P$-value \\
\hline Surgical site infection & 0.760 & $0.269-2.152$ & 0.605 & 0.633 & $0.161-2.489$ & 0.513 \\
Anastomotic leakage & 1.019 & $0.390-2.663$ & 0.970 & 1.139 & $0.313-4.140$ & 0.844 \\
Pneumonia & 2.952 & $1.118-7.798$ & 0.029 & 3.003 & $1.117-8.072$ & 0.029 \\
\hline
\end{tabular}

OR: odds ratio; CI: confidence interval.

Table 6. Perioperative risk factors for pneumonia in patients undergoing esophageal cancer surgery.

\begin{tabular}{cccccccc}
\hline Variable & & Unadjusted OR & $95 \% \mathrm{CI}$ & $P$-value & Adjusted OR & $95 \% \mathrm{CI}$ & $P$-value \\
\hline Albumin & Pre & 0.196 & $0.053-0.732$ & 0.015 & 0.189 & $0.047-0.759$ & 0.02 \\
Neutrophil-lymphocyte ratio & Pre & 1.228 & $1.039-1.452$ & 0.016 & 1.229 & $1.035-1.460$ & 0.02 \\
\hline
\end{tabular}

OR: odds ratio; CI: confidence interval; Pre: preoperative; Post: postoperative.

\section{Conclusion}

Perioperative factors related to cancer recurrence and 2-year survival were postoperative pneumonia, which was highly linked to preoperative lower albumin level and higher neutrophil-lymphocyte ratio. Intraoperative use of opioids was not correlated with postoperative complications, cancer recurrence, or 2-year survival. Cancer stage and preoperative physical status including immune and nutrient state may be more important for postoperative prognosis after esophageal cancer surgery. Therefore, opioids should be used as a component of balanced anesthesia for esophageal cancer surgery.

\section{Acknowledgements}

We thank Kelly Zammit, BVSc, and Alison Sherwin, PhD, from Edanz Group (http://www.edanzediting.com/ac), for editing a draft of this manuscript.

This work was funded by Grants-in-Aid for Scientific Research from the Japan Society for the Promotion of Science, Tokyo, Japan (to Maiko Hasegawa-Moriyama).

\section{Ethical Approval and Consent to Participate}

This study was approved by the Ethics Committee of Kagoshima University Hospital. Because of the anonymous retrospective nature of this study, requirement of informed consent was waived.

\section{Availability of Data and Materials}

The datasets analyzed during the current study are available from the corresponding author on the request.

\section{Conflicts of Interest}

The author declares no conflicts of interest regarding the publication of this paper. 


\section{Authors' Contributions}

M. H. M. contributed to the study conception and design, acquisition of data, and analysis and interpretation of data; M. H. M. and Y. K. contributed to drafting of the article.

\section{References}

[1] Bray, F., Ferlay, J., Soerjomataram, I., Siegel, R.L., Torre, L.A. and Jemal, A. (2018) Global Cancer Statistics 2018: Globocan Estimates of Incidence and Mortality Worldwide for 36 Cancers in 185 Countries. CA: A Cancer Journal for Clinicians, 68, 394-424. https://doi.org/10.3322/caac.21492

[2] Matsuda, S., Takeuchi, H., Kawakubo, H., et al. (2015) Correlation between Intense Postoperative Inflammatory Response and Survival of Esophageal Cancer Patients Who Underwent Transthoracic Esophagectomy. Annals of Surgical Oncology, 22, 4453-4460. https://doi.org/10.1245/s10434-015-4557-5

[3] Thomson, I.G., Smithers, B.M., Gotley, D.C., et al. (2010) Thoracoscopic-Assisted Esophagectomy for Esophageal Cancer: Analysis of Patterns and Prognostic Factors for Recurrence. Annals of Surgery, 252, 281-291. https://doi.org/10.1097/SLA.0b013e3181e909a2

[4] Liu, Z., Jiang, M., Zhao, J. and Ju, H. (2007) Circulating Tumor Cells in Perioperative Esophageal Cancer Patients: Quantitative Assay System and Potential Clinical Utility. Clinical Cancer Research, 13, 2992-2997. https://doi.org/10.1158/1078-0432.CCR-06-2072

[5] Hsu, P.K., Wang, B.Y., Huang, C.S., Wu, Y.C. and Hsu, W.H. (2011) Prognostic Factors for Post-Recurrence Survival in Esophageal Squamous Cell Carcinoma Patients with Recurrence after Resection. Journal of Gastrointestinal Surgery, 15, 558-565. https://doi.org/10.1007/s11605-011-1458-1

[6] Shapiro, J., van Lanschot, J.J.B., Hulshof, M., et al. (2015) Neoadjuvant Chemoradiotherapy plus Surgery versus Surgery alone for Oesophageal or Junctional Cancer (Cross): Long-Term Results of a Randomised Controlled Trial. The Lancet Oncology, 16, 1090-1098. https://doi.org/10.1016/S1470-2045(15)00040-6

[7] Tan, J.T., Yang, Y., Mao, N.Q., et al. (2017) Effect of Neoadjuvant Chemoradiotherapy on Perioperative Immune Function of Patients with Locally Advanced Esophageal Cancer. Diseases of the Esophagus, 30, 1-8. https://doi.org/10.1111/dote.12486

[8] Ben-Eliyahu, S., Page, G.G., Yirmiya, R. and Shakhar, G. (1999) Evidence that Stress and Surgical Interventions Promote Tumor Development by Suppressing Natural Killer Cell Activity. International Journal of Cancer, 80, 880-888. https://doi.org/10.1002/(SICI)1097-0215(19990315)80:6<880::AID-IJC14>3.0.CO;2-Y

[9] Cata, J.P., Wang, H., Gottumukkala, V., Reuben, J. and Sessler, D.I. (2013) Inflammatory Response, Immunosuppression, and Cancer Recurrence after Perioperative Blood Transfusions. British Journal of Anaesthesia, 110, 690-701. https://doi.org/10.1093/bja/aet068

[10] Yeager, M.P., Colacchio, T.A., Yu, C.T., et al. (1995) Morphine Inhibits Spontaneous and Cytokine-Enhanced Natural Killer Cell Cytotoxicity in Volunteers. Anesthesiology, 83, 500-508. https://doi.org/10.1097/00000542-199509000-00008

[11] Brand, J.M., Kirchner, H., Poppe, C. and Schmucker, P. (1997) The Effects of General Anesthesia on Human Peripheral Immune Cell Distribution and Cytokine 
Production. Clinical Immunology and Immunopathology, 83, 190-194. https://doi.org/10.1006/clin.1997.4351

[12] Jun, I.J., Jo, J.Y., Kim, J.I., et al. (2017) Impact of Anesthetic Agents on Overall and Recurrence-Free Survival in Patients Undergoing Esophageal Cancer Surgery: A Retrospective Observational Study. Scientific Reports, 7, 14020. https://doi.org/10.1038/s41598-017-14147-9

[13] Perez-Gonzalez, O., Cuellar-Guzman, L.F., Navarrete-Pacheco, M., Ortiz-Martinez, J.J., Williams, W.H. and Cata, J.P. (2018) Impact of Regional Anesthesia on Gastroesophageal Cancer Surgery Outcomes: A Systematic Review of the Literature. Anesthesia \& Analgesia, 127, 753-758. https://doi.org/10.1213/ANE.0000000000003602

[14] Oh, T.K., Kim, K., Jheon, S.H., et al. (2018) Long-Term Oncologic Outcomes, Opioid Use, and Complications after Esophageal Cancer Surgery. Journal of Clinical Medicine, 7, 33. https://doi.org/10.3390/jcm7020033

[15] Sekandarzad, M.W., van Zundert, A.A.J., Lirk, P.B., Doornebal, C.W. and Hollmann, M.W. (2017) Perioperative Anesthesia Care and Tumor Progression. Anesthesia \& Analgesia, 124, 1697-1708. https://doi.org/10.1213/ANE.0000000000001652

[16] Du, K.N., Feng, L., Newhouse, A., et al. (2018) Effects of Intraoperative Opioid Use on Recurrence-Free and Overall Survival in Patients with Esophageal Adenocarcinoma and Squamous Cell Carcinoma. Anesthesia \& Analgesia, 127, 210-216. https://doi.org/10.1213/ANE.0000000000003428

[17] Bartal, I., Melamed, R., Greenfeld, K., et al. (2010) Immune Perturbations in Patients along the Perioperative Period: Alterations in Cell Surface Markers and Leukocyte Subtypes before and after Surgery. Brain, Behavior, and Immunity, 24, 376-386. https://doi.org/10.1016/j.bbi.2009.02.010

[18] Iwata, E., Shigematsu, H., Okuda, A., et al. (2016) Lymphopenia at 4 Days Postoperatively Is the Most Significant Laboratory Marker for Early Detection of Surgical site Infection Following Posterior Lumbar Instrumentation Surgery. Asian Spine Journal, 10, 1042-1046. https://doi.org/10.4184/asj.2016.10.6.1042

[19] Silberman, S., Abu-Yunis, U., Tauber, R., et al. (2018) Neutrophil-Lymphocyte Ratio: Prognostic Impact in Heart Surgery. Early Outcomes and Late Survival. Annals of Thoracic Surgery, 105, 581-586. https://doi.org/10.1016/j.athoracsur.2017.07.033

[20] Walsh, S.R., Cook, E.J., Goulder, F., Justin, T.A. and Keeling, N.J. (2005) Neutrophil-Lymphocyte Ratio as a Prognostic Factor in Colorectal Cancer. Journal of Surgical Oncology, 91, 181-184. https://doi.org/10.1002/jso.20329

[21] Forget, P., Machiels, J.P., Coulie, P.G., et al. (2013) Neutrophil: Lymphocyte Ratio and Intraoperative Use of Ketorolac or Diclofenac Are Prognostic Factors in Different Cohorts of Patients Undergoing Breast, Lung, and Kidney Cancer Surgery. Annals of Surgical Oncology, 20, S650-S660. https://doi.org/10.1245/s10434-013-3136-x

[22] Shinoda, T., Murakami, W., Takamichi, Y., Iizuka, H., Tanaka, M. and Kuwasako, Y. (2013) Effect of Remifentanil Infusion Rate on Stress Response in Orthopedic Surgery Using a Tourniquet Application. BMC Anesthesiology, 13, 14. https://doi.org/10.1186/1471-2253-13-14

[23] Kurosawa, S. (2012) Anesthesia in Patients with Cancer Disorders. Current Opinion in Anaesthesiology, 25, 376-384. https://doi.org/10.1097/ACO.0b013e328352b4a8

[24] Wiese, A.D., Griffin, M.R., Schaffner, W., et al. (2018) Opioid Analgesic Use and Risk for Invasive Pneumococcal Diseases: A Nested Case-Control Study. Annals of 
Internal Medicine, 168, 396-404. https://doi.org/10.7326/M17-1907

[25] Hofbauer, R., Frass, M., Gmeiner, B., et al. (2000) Effects of Remifentanil on Neutrophil Adhesion, Transmigration, and Intercellular Adhesion Molecule Expression. Acta Anaesthesiologica Scandinavica, 44, 1232-1237. https://doi.org/10.1034/j.1399-6576.2000.441008.x

[26] Cronin, A.J., Aucutt-Walter, N.M., Budinetz, T., et al. (2003) Low-Dose Remifentanil Infusion Does Not Impair Natural Killer Cell Function in Healthy Volunteers. British Journal of Anaesthesia, 91, 805-809. https://doi.org/10.1093/bja/aeg273

[27] Boland, J.W. and Pockley, A.G. (2018) Influence of Opioids on Immune Function in Patients with Cancer Pain: From Bench to Bedside. British Journal of Pharmacology, 175, 2726-2736. https://doi.org/10.1111/bph.13903

\section{Abbreviations}

FE: Fentanyl Equivalents

SSI: Surgical Site Infection

OR: Odds Ratio

CI: Confidence Interval 